\title{
Quasiprobability distribution functions for finite-dimensional discrete phase spaces: Spin-tunneling effects in a toy model
}

\author{
Marcelo A. Marchiolli, ${ }^{*}$ Evandro C. Silva, ${ }^{\dagger}$ and Diógenes Galetti ${ }^{\ddagger}$ \\ Instituto de Física Teórica, Universidade Estadual Paulista, Rua Pamplona 145, 01405-900, São Paulo, SP, Brazil
}

(Received 19 July 2008; published 17 February 2009)

\begin{abstract}
We show how quasiprobability distribution functions defined over $N^{2}$-dimensional discrete phase spaces can be used to treat physical systems described by a finite space of states which exhibit spin-tunneling effects. This particular approach is then applied to the Lipkin-Meshkov-Glick model in order to obtain the time evolution of the discrete Husimi function, and as a by-product the energy gap for a symmetric combination of ground and first excited states. Moreover, we also show how an angle-based potential approach can be efficiently employed to explain qualitatively certain features of the energy gap in terms of a spin tunneling. Entropy functionals are also discussed in this context. Such results reinforce not only the formalism per se but also the possibility of some future potential applications in other branches of physics.
\end{abstract}

DOI: 10.1103/PhysRevA.79.022114

PACS number(s): 03.65.Ca, 03.65.Xp, 21.60.Fw

\section{INTRODUCTION}

In the last decades, much effort has been devoted to characterize quantum tunneling processes in mesoscopic and/or macroscopic systems, emphasizing the importance of the degree of freedom related to the angular momentum and angle pair [1]. In what concerns the spin tunneling, certain theoretical approaches have pointed to some different ways of treating this problem, each one presenting a particular set of convenient inherent mathematical properties [2]. From this perspective, if one considers physical systems with a finitedimensional space of states and described by discrete variables, a sound theoretical framework must be employed to characterize properly such nonclassical effect. In fact, an alternative approach to the system description of these specific cases can be pointed out. First, we recognize that the state spaces associated with those particular physical systems are $N$-dimensional Hilbert spaces. Next, in connection with these finite Hilbert spaces, it should be stressed that quantum representations of $N^{2}$-dimensional discrete phase spaces can also be constructed [3]. Thus, relevant operators whose kinematical and/or dynamical contents carry all the necessary information for describing those quantum systems can now be promptly mapped in such phase spaces. In this sense, although there are various phase space in front of formalisms proposed in the literature for treating finite-dimensional physical systems [4], let us focus our attention upon the framework developed in Refs. [5-9] for the discrete representatives of the quasiprobability distribution functions defined in $N^{2}$-dimensional phase spaces, which has its algebraic structure based on the technique of constructing unitary operator bases initially formulated by Schwinger [10]. The virtue of this discrete quantum phase-space approach is that it allows us to exhibit and handle the pair of complementary variables related to a particular degree of freedom we are dealing with, as well as to recognize the quantum correla-

\footnotetext{
*mamarchi@ift.unesp.br

†mandrake@ift.unesp.br

†galetti@ift.unesp.br
}

tions between them. The basic idea then consists in exploring this mathematical tool in order to study those quantum correlations in connection with spin-tunneling processes. Therefore, besides having the energy spectrum, which can be obtained via direct diagonalization of the Hamiltonian system, we can also have additional quantum information about the physical system through the study of the corresponding discrete Wigner and/or Husimi functions.

Such theoretical framework is then applied, in particular, to the Lipkin-Meshkov-Glick (LMG) model [11], which was originally introduced over 40 years ago in nuclear physics [12] for treating certain fermionic systems. This important toy model has, since then, been extensively studied in the literature because of its apparent simplicity [13]. Indeed, it can also be viewed as a finite set of spins half-mutually interacting in the $x y$ plane subjected to a transverse magnetic field [14]. Moreover, our interest in this model also resides in the fact that spin tunneling can be considered to occur.

In this work, we show how the time-dependent discrete Husimi function and an angle-based potential description [15-17] can be combined in order to describe a group of physical processes that encompasses, among other things, the spin-tunneling effects. This particular approach leads us not only to extract the energy gap for a symmetric combination of ground and first excited states, but also to corroborate its inherent applicability to analogous physical systems such as in magnetic molecules [18].

This paper is organized as follows. In Sec. II, we present a condensed review of the theoretical apparatus used to describe $N^{2}$-dimensional phase spaces and also discuss some essential features exhibited by the time-dependent discrete Husimi function. In Sec. III, we apply our results to the LMG model to explore qualitatively the spin-tunneling effects for a symmetric combination of ground and first excited states. Finally, Sec. IV contains our summary and conclusions.

\section{THEORETICAL APPARATUS FOR FINITE-DIMENSIONAL PHASE SPACES}

Our theoretical framework is totally based upon the formalism developed in Refs. [7,8] for physical systems with 
finite-dimensional space of states. In this sense, let us introduce some basic elements which represent our guidelines for the fundamentals of the formal description of $N^{2}$-dimensional phase spaces by means of discrete variables. The first important element is the $\bmod (N)$-invariant operator basis

$$
\mathbf{T}^{(s)}(\mu, \nu)=\frac{1}{\sqrt{N}} \sum_{\eta, \xi=-\ell}^{\ell} \exp \left[-\frac{2 \pi i}{N}(\eta \mu+\xi \nu)\right] \mathbf{S}^{(s)}(\eta, \xi),
$$

which consists of a discrete Fourier transform of the extended mapping kernel $\mathbf{S}^{(s)}(\eta, \xi)=[\mathcal{K}(\eta, \xi)]^{-s} \mathbf{S}(\eta, \xi)$,

$$
\mathbf{S}(\eta, \xi):=\frac{1}{\sqrt{N}} \exp \left(\frac{i \pi}{N} \eta \xi\right) \mathbf{U}^{\eta} \mathbf{V}^{\xi}
$$

being the symmetrized version of the unitary operator basis proposed by Schwinger [10]. In this particular approach, the labels $\eta$ and $\xi$ are associated with the dual momentum and coordinatelike variables of a discrete $N^{2}$-dimensional phase space. Note that these labels are congruent modulo $N$ and assume integer values in the symmetrical interval $[-\ell, \ell]$ for $\ell=(N-1) / 2$ fixed. Besides, the extra term $\mathcal{K}(\eta, \xi)$ is defined through the ratio $\mathcal{M}(\eta, \xi) / \mathcal{M}(0,0)$, where the function [7]

$$
\begin{aligned}
\mathcal{M}(\eta, \xi)= & \frac{\sqrt{\mathfrak{a}}}{2}\left\{\vartheta_{3}(\mathfrak{a} \eta \mid i \mathfrak{a})\left[\vartheta_{3}(\mathfrak{a} \xi \mid i \mathfrak{a})+\vartheta_{4}(\mathfrak{a} \xi \mid i \mathfrak{a}) e^{i \pi \eta}\right]\right. \\
& \left.+\vartheta_{4}(\mathfrak{a} \eta \mid i \mathfrak{a})\left[\vartheta_{3}(\mathfrak{a} \xi \mid i \mathfrak{a}) e^{i \pi \xi}+\vartheta_{4}(\mathfrak{a} \xi \mid i \mathfrak{a}) e^{i \pi(\eta+\xi+N)}\right]\right\}
\end{aligned}
$$

with $\mathfrak{a}=(2 N)^{-1}$ is responsible for the sum of products of Jacobi $\vartheta$ functions evaluated at integer arguments, and $s$ refers to a complex parameter that satisfies the relation $|s|$ $\leqslant 1$. It is worth mentioning that a compilation of results and properties which characterize the algebraic structure of the discrete mapping kernel $\mathbf{T}^{(s)}(\mu, \nu)$, as well as the unitary operators $\mathbf{U}$ and $\mathbf{V}$, can be promptly found in Refs. [5,7]. For physical applications related to quantum tomography and quantum teleportation, see also Ref. [8].

Next, let us assume that $\boldsymbol{\rho}(t)$ reflects the dynamics of a particular quantum system characterized by a finitedimensional space of states. The one-to-one mapping between density operators and functions belonging to an $N^{2}$-dimensional phase space labeled by $\{\mu, \nu\} \in[-\ell, \ell]$ is attained, in our description, by means of the parametrized function $F^{(s)}(\mu, \nu ; t):=\operatorname{Tr}\left[\mathbf{T}^{(s)}(\mu, \nu) \boldsymbol{\rho}(t)\right]$. Expressed as a double discrete Fourier transform of the discrete $s$-ordered characteristic function $\Xi^{(s)}(\eta, \xi ; t):=\operatorname{Tr}\left[\mathbf{S}^{(s)}(\eta, \xi) \boldsymbol{\rho}(t)\right]$, it has a well-established continuous counterpart within the CahillGlauber formalism [19]. Moreover, for $s=-1,0,1$ the timedependent function $F^{(s)}(\mu, \nu ; t)$ is directly related to the respective discrete Husimi, Wigner, and Glauber-Sudarshan distribution functions. An interesting formal result from this formalism explores the connection between discrete Wigner and Husimi functions-here denoted by $\mathcal{W}(\mu, \nu ; t)$ and $\mathcal{H}(\mu, \nu ; t)$ - through the equation

$$
\mathcal{H}(\mu, \nu ; t)=\frac{1}{N} \sum_{\mu^{\prime}, \nu^{\prime}=-\ell}^{\ell} E\left(\mu, \nu \mid \mu^{\prime}, \nu^{\prime}\right) \mathcal{W}\left(\mu^{\prime}, \nu^{\prime} ; t\right),
$$

where $E\left(\mu, \nu \mid \mu^{\prime}, \nu^{\prime}\right) \equiv \operatorname{Tr}\left[\mathbf{T}^{(0)}(\mu, \nu) \mathbf{T}^{(-1)}\left(\mu^{\prime}, \nu^{\prime}\right)\right]$ defines a smoothing process characterized by a discrete phase-space function that closely resembles the role of a Gaussian function in the continuous phase space. In fact, Eq. (3) represents an intermediate smoothing sequence within a hierarchical process among the quasiprobability distribution functions in finite-dimensional spaces [8]. In what concerns the time evolution of the density operator, it must be stressed that $\boldsymbol{\rho}(t)$ satisfies the von Neumann-Liouville equation and its corresponding mapped expression in the discrete phase-space representation leads us to obtain a differential equation for $\mathcal{W}(\mu, \nu ; t)$, whose solution was explicitly determined in Ref. [6]. Hence, the discrete Husimi function can now be immediately inferred.

Now, let us establish an important mathematical result for the time-dependent discrete Husimi functions. First, it should be noticed that $\mathcal{H}(\mu, \nu ; t)$ is strictly positive and limited to the interval $[0,1]$ for any $t \geqslant 0$; second, the phase space treated here consists of a finite mesh with $N^{2}$ points and characterized by the discrete variables $\mu$ and $\nu$. So, the discrete Husimi function can be mapped onto a real $N \times N$ matrix $H(t)$ whose elements $\left[h_{r s}(t)\right]_{r, s=1, \ldots, N}$ obey two essential properties that imply in the conservation of probabilities on a discrete phase space, that is,

$$
0 \leqslant h_{r s}(t) \leqslant 1 \quad \text { and } \sum_{r, s=1}^{N} h_{r s}(t)=1
$$

The interaction with any dissipative environment is automatically discarded within this context. Such mathematical procedure brings some operational advantages in our description since the matrix $H(t)$ can be promptly diagonalized onto the eigenspaces,

$$
V_{\lambda_{i}}=\left\{V_{i} \in V: \mathbb{H V} V_{i}=\lambda_{i} V_{i}\right\}
$$

characterized by the eigenvectors $\left\{\mathrm{V}_{i}(t)\right\}_{i=1, \ldots, N}$ and their corresponding eigenvalues $\left\{\lambda_{i}(t)\right\}_{i=1, \ldots, N}$ for $t$ fixed. It is worth emphasizing that the eigenvalues obtained from this particular diagonalization process assume, in general, both real and complex values, and this fact can be explained by means of matrix analysis [20]. A pertinent question then emerges from our considerations on $N^{2}$-dimensional phase spaces: "Can both real and complex eigenvalues be associated with some physical process?"

To answer this question, let us initially decompose the matrix $H(t)$ as a sum of two Hermitian and anti-Hermitian $N \times N$ matrices, namely, $\mathbb{H}(t)=\mathrm{A}(t)+\mathrm{B}(t)$, both matrices being constructed out following the mathematical recipe $A(t)$ $=\frac{1}{2}\left[H(t)+H^{\dagger}(t)\right]$ and $\mathbb{B}(t)=\frac{1}{2}\left[H(t)-H^{\dagger}(t)\right]$. In this situation, the diagonalization process attributes real eigenvalues for $\mathrm{A}(t)$, while $\mathbb{B}(t)$ has eigenvalues which are pure imaginary (or zero). Besides, the trace of $H(t)$ is preserved, i.e., 


$$
\operatorname{Tr}[\mathbb{H}(t)] \equiv \operatorname{Tr}[\mathrm{A}(t)]=\sum_{i=1}^{N} \sigma_{A}^{(i)}(t) .
$$

Here, $\left\{\sigma_{\mathrm{A}}^{(i)}(t)\right\}_{i=1, \ldots, N}$ and $\left\{\sigma_{\mathrm{B}}^{(i)}(t)\right\}_{i=1, \ldots, N}$ represent the respective eigenvalues of the matrices $\mathrm{A}(t)$ and $\mathrm{B}(t)$ for all $t \geqslant 0$; therefore, it is easy to verify that these eigenvalues are now responsible for the real and imaginary parts of the complex eigenvalues $\left\{\lambda_{i}(t)\right\}_{i=1, \ldots, N}$. Next, let us introduce an auxiliary tool characterized by the entropy functional

$$
E\left[\left\{\lambda_{i}\right\} ; t\right]=-\sum_{i=1}^{N}\left|\lambda_{i}(t)\right| \ln \left[\left|\lambda_{i}(t)\right|\right],
$$

which allows us to infer all the different contributions associated with $\left\{\lambda_{i}(t)\right\} \in \mathrm{C}$. From the operational point of view, the simplicity of this measure represents an effective gain to our task since the diagonalization process of the timedependent discrete Husimi function is sufficient in this case for determining $E\left[\left\{\lambda_{i}\right\} ; t\right]$. The next step then consists in considering a well-known physical system that leads us to find out any concrete evidence in (4) of some particular physical process inherent to the model. In this sense, we will apply the theoretical framework here discussed to a solvable quasispin model whose Hamiltonian, although simple, presents some interesting physical and mathematical features, namely, the Lipkin-Meshkov-Glick (LMG) model [11].

\section{LMG MODEL}

Originally proposed with the intent of testing mean-field approximations in many-body systems, the LMG model is here introduced through the Hamiltonian [11]

$$
\mathbf{H}=\frac{\epsilon}{2} \sum_{\mathfrak{q}, \sigma} \sigma \mathbf{a}_{\mathfrak{q}, \sigma}^{\dagger} \mathbf{a}_{\mathfrak{q}, \sigma}+\frac{V}{2} \sum_{\mathfrak{q}, \mathfrak{q}^{\prime}, \sigma} \mathbf{a}_{\mathfrak{q}, \sigma}^{\dagger} \mathbf{a}_{\mathfrak{q}^{\prime}, \sigma}^{\dagger} \mathbf{a}_{\mathfrak{q}^{\prime},-\sigma} \mathbf{a}_{\mathfrak{q},-\sigma}
$$

which describes a collection of $N_{p}$ fermions distributed in two $N_{p}$-fold degenerate levels separated by an energy $\epsilon$. The degenerate states within each level are labeled in this expression by means of the quantum numbers $\mathfrak{q} \in\left[1, N_{p}\right]$ and $\sigma$ $= \pm 1(+1$ and -1 represent the respective higher and lower levels), $N_{p}$ being considered an even number.

It is worth noticing that the introduction of the quasispin operators [12]

$$
\mathbf{J}_{ \pm}:=\sum_{\mathfrak{q}} \mathbf{a}_{\mathfrak{q}, \pm 1}^{\dagger} \mathbf{a}_{\mathfrak{q}, \mp 1} \quad \text { and } \mathbf{J}_{\mathfrak{z}}:=\frac{1}{2} \sum_{\mathfrak{q}, \sigma} \sigma \mathbf{a}_{\mathfrak{q}, \sigma}^{\dagger} \mathbf{a}_{\mathfrak{q}, \sigma}
$$

into the LMG Hamiltonian not only reveals its underlying $\mathrm{SU}(2)$ structure, since the operators $\mathbf{J}_{ \pm}$and $\mathbf{J}_{\mathfrak{z}}$ obey the standard commutation relations $\left[\mathbf{J}_{+}, \mathbf{J}_{-}\right]=2 \mathbf{J}_{\mathfrak{z}}$ and $\left[\mathbf{J}_{\mathfrak{z}}, \mathbf{J}_{ \pm}\right]$ $= \pm \mathbf{J}_{ \pm}$, but also allows us to treat collective excitations of the fermionic system in a more suitable form, namely, $\mathbf{H}$ $=\epsilon \mathbf{J}_{\mathfrak{z}}+(V / 2)\left(\mathbf{J}_{+}^{2}+\mathbf{J}_{-}^{2}\right)$. Indeed, the $\mathbf{J}_{\mathfrak{z}}$ term of this Hamiltonian operator gives one-half the difference of the number of particles laid on the upper and lower levels, while the second term, involving the operators $\mathbf{J}_{+}^{2}$ and $\mathbf{J}_{-}^{2}$, is associated with the interaction between a pair of particles located at the same energy level, being also responsible for the scattering process of this pair to the other level where the quantum number $\mathfrak{q}$ of each particle is preserved. For simplicity, let us rewrite such Hamiltonian operator in order to scale the interaction term to the particle number while measuring the energy in terms of $\epsilon$, i.e.,

$$
\mathbf{H}_{L}:=\frac{\mathbf{H}}{\epsilon}=\mathbf{J}_{\mathfrak{z}}+\frac{\chi}{2 N_{p}}\left(\mathbf{J}_{+}^{2}+\mathbf{J}_{-}^{2}\right)
$$

with $\chi=N_{p} V / \epsilon$. Since $\left[\mathbf{H}_{L}, \mathbf{J}^{2}\right]=0$, it turns immediate to see that $\mathbf{H}_{L}$ can be diagonalized within each $(2 J+1)$-dimensional multiplet labeled by the eigenvalues of $\mathbf{J}^{2}$ and $\mathbf{J}_{\mathfrak{z}}$, which accounts for the soluble character of the associated quantum model $[11,12]$. It is worth stressing that the ground state belongs to the finite multiplet characterized by $J=\frac{N_{p}}{2}$ $=\max \left(J_{\mathfrak{z}}\right)$, so that its $\mathbf{J}^{2}$ and $\mathbf{J}_{\mathfrak{z}}$ quantum numbers are $\left(\frac{N_{p}^{2}}{2}\right)$ $\left(\frac{N_{p}}{2}+1\right)$ and $-\frac{N_{p}}{2}$, respectively. Hereafter, we will be interested only in this particular multiplet containing the ground state and whose dimension is given by $N=2 J+1=N_{p}+1$. This $\left(N_{p}+1\right)$-dimensional multiplet will be considered as our underlying Hilbert space of interest [21], and also will be used to construct the $\left(N_{p}+1\right)^{2}$-dimensional discrete phase space.

Next, let us mention some few words about two discrete conserved quantities inherent to the LMG model which reflect certain symmetry properties. The simplest operator commuting with the Hamiltonian (5), therefore giving a constant of motion, is the parity operator $\Pi:=\exp \left(i \pi \mathbf{J}_{\mathfrak{z}}\right)$. This fact tells us that the Hamiltonian matrix, in the $\mathbf{J}_{\mathfrak{z}}$ representation, breaks into two disjoint blocks involving only even and odd eigenvalues of $\mathbf{J}_{\mathfrak{z}}$, respectively. The second interesting quantity comes from the anticommutation relation $\left\{\mathbf{H}_{L}, \mathbf{R}\right\}=0$, where

$$
\mathbf{R}(-\pi / 2, \pi, 0):=\exp \left[i(\pi / 2) \mathbf{J}_{\mathfrak{z}}\right] \exp \left(i \pi \mathbf{J}_{y}\right)
$$

corresponds to a rotation of the angular momentum quantization frame by the Euler angles $(-\pi / 2, \pi, 0)$, thus transforming $\mathbf{H}_{L} \rightarrow-\mathbf{H}_{L}$. In this case, if $\left|E_{j}\right\rangle$ is an energy eigenstate with eigenvalue $E_{j}$, then $\mathbf{R}\left|E_{j}\right\rangle$ is also an eigenstate of $\mathbf{H}_{L}$ with eigenvalue $-E_{j}$. This symmetry property of the Hamiltonian operator (5) gives rise to an energy spectrum that is symmetric about zero.

After this condensed review, let us establish a sequence of steps that permits us to evaluate the time evolution of the discrete Husimi function for the LMG model. The first one consists in adopting the theoretical approach developed in Ref. [15] for the time-dependent discrete Wigner function $\mathcal{W}(m, n ; t)$ defined upon an $N^{2}$-dimensional phase space labeled by the angular momentum and angle pair $(m, n)$ $\in\left[-N_{p} / 2, N_{p} / 2\right]$. Since this approach depends on the Wigner function evaluated at time $t=0$, the second one consists in fixing the initial state as a symmetric combination of the ground and first excited states following the recipe described in [16]. The next and last step refers to the smoothing process given by Eq. (3), which leads us to finally obtain the desired result. Figure 1 shows the three-dimensional plots of $\mathcal{H}(m, n ; \tau)$ versus $(m, n) \in[-10,10]$ with $N_{p}=20$, and $\chi$ $=1.5$ fixed. In the numerical investigations, we have adopted some specific values for the dimensionless time $\tau$ in order to 


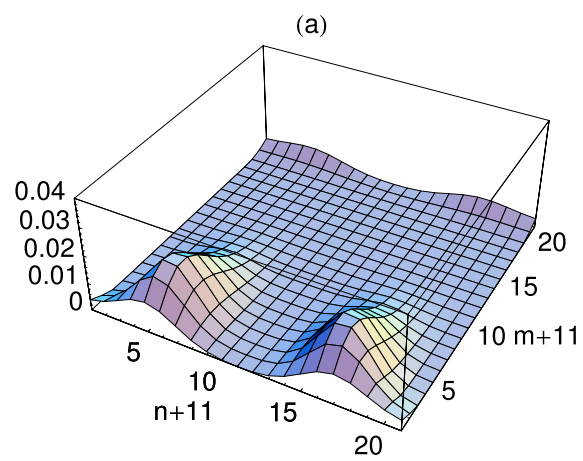

(b)

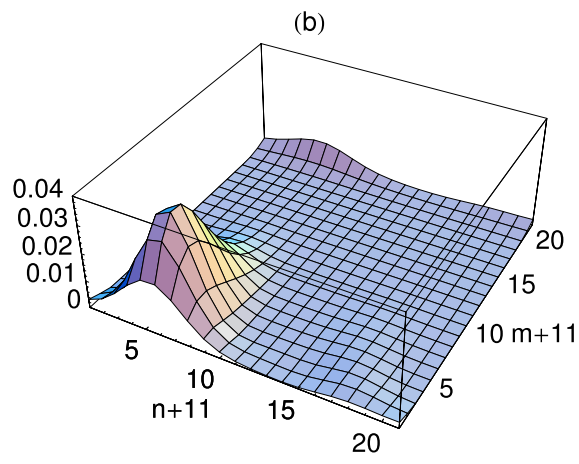

(c)

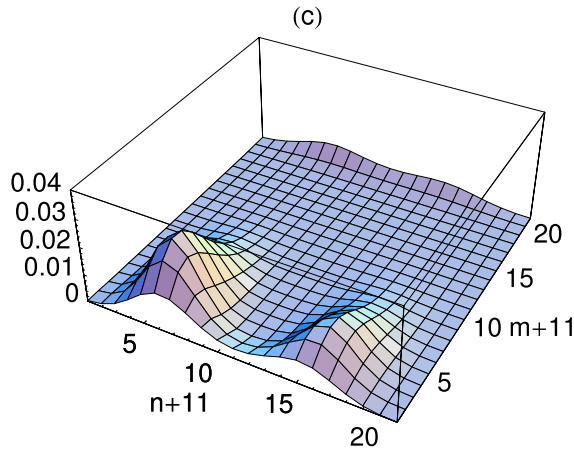

(d)

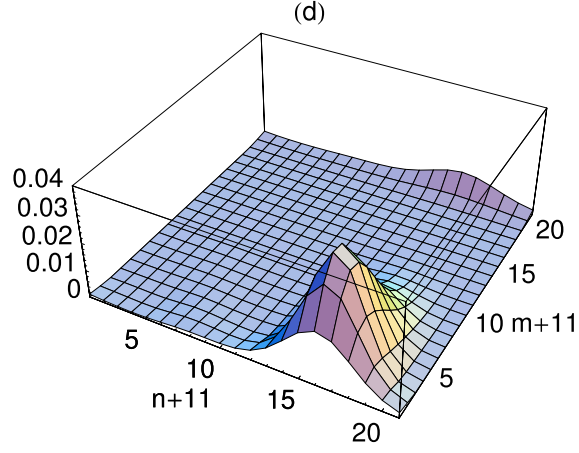

FIG. 1. (Color online) Time evolution of $\mathcal{H}(m, n ; \tau)$ for the Lipkin-Meshkov-Glick model with $N_{p}=20$ and $\chi=1.5$ fixed, where the labels $m$ and $n$ characterize, respectively, the dimensionless angular momentum and angle pair. These pictures show, in particular, how the two-body interaction term present in the Hamiltonian $\mathbf{H}$ affects the initial distribution $\mathcal{H}(m, n ; 0)$ for different values of dimensionless time $\tau$. We have adopted in our numerical investigations the values (a) $\tau=0$, (b) $\tau \approx 6.5$, (c) $\tau \approx 15.9$, and (d) $\tau \approx 25.3$, which illustrate a representative but not complete evolution of the discrete Husimi function.

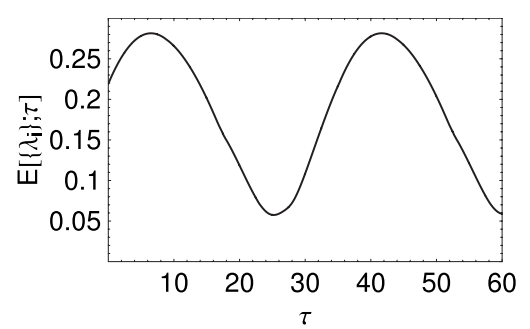

FIG. 2. Time evolution of $E\left[\left\{\lambda_{i}\right\} ; \tau\right]$ versus $\tau \in[0,60]$ for the same set of parameters used in Fig. 1. It is worth noticing that the maximum and minimum points of this curve correspond to the cases when the discrete Husimi function reaches its peaks in the respective negative and positive regions of the angle sector of the finite phase space. This fact permits us to estimate the energy gap $\Delta=\left(E_{1}-E_{0}\right) / \epsilon$ from our numerical data, namely, $\Delta \approx 0.1784$, which corroborates the value obtained through the diagonalization process of the Hamiltonian operator (5) in the $\mathbf{J}_{\mathfrak{z}}$ basis $(\Delta=0.1788)$.

illustrate the effects of the two-body interaction term in the original Hamiltonian $\mathbf{H}$ (or, equivalently, the second term of $\mathbf{H}_{L}$ constituted by the operators $\mathbf{J}_{+}^{2}$ and $\mathbf{J}_{-}^{2}$ ) on a given initial configuration of the finite phase space. Thus, Fig. 1(a) represents the discrete Husimi function $\mathcal{H}(m, n ; 0)$ which reflects the initial condition of the model under investigation, that is, it shows two distinct regions equally distributed in the $21^{2}$-dimensional phase space of the particular two-level system. Figure 1(b) corresponds to a subsequent time $\tau \approx 6.5$, where we perceive that the probability distribution was almost totally reallocated in one side of the discrete phase space, which means that both the angular momentum and angle components present negative values. By its turn, Fig. 1(c) shows an intermediate process for $\tau \approx 15.9$ and quite similar to that found in (a) when $\tau=0$. Finally, Fig. 1(d) illustrates the migration, in $\tau \approx 25.3$, to a distribution of positive values of angle components in constrast to that viewed in Fig. 1(b). Note that, in particular, the quantum dynamics of this system inhibits a distribution function centered at $n$ $=0$.

Although the periodic pattern verified for the discrete $\mathrm{Hu}$ simi function can be explained, in principle, through the periodic fluctuation of particle populations between the two energy levels, it is not clear until now its relation with the energy gap $\Delta=\left(E_{1}-E_{0}\right) / \epsilon$, associated with the quasispin tunneling occuring in the present situation, as well as its dependence on the interaction parameter $\chi$. To clarify this point, some considerations on the energy spectrum related to $\mathbf{H}_{L}$ deserve to be properly mentioned: (i) It is numerically obtained by just diagonalizing the matrix associated with the Hamiltonian (5) in the $\mathbf{J}_{3}$ basis (for more details on the exact solutions of the LMG model, see Ref. [11]); as a direct consequence of this result, (ii) the energy gap curve shows an explicit dependence on $\chi$, that is, as the two-body correlation strength increases, the energy gap decreases $[11,16]$. Therefore, if one changes the interaction parameter $\chi$ (or the energy gap $\Delta)$, the periodic pattern showed by $\mathcal{H}(m, n ; \tau)$ will be also modified. Next, with the help of the entropy functional (4), we will estimate this quantity for the same set of parameters used in the previous figure. In this way, Fig. 2 shows the plot of $E\left[\left\{\lambda_{i}\right\} ; \tau\right]$ versus $\tau \in[0,60]$ for $N_{p}=20$ and 

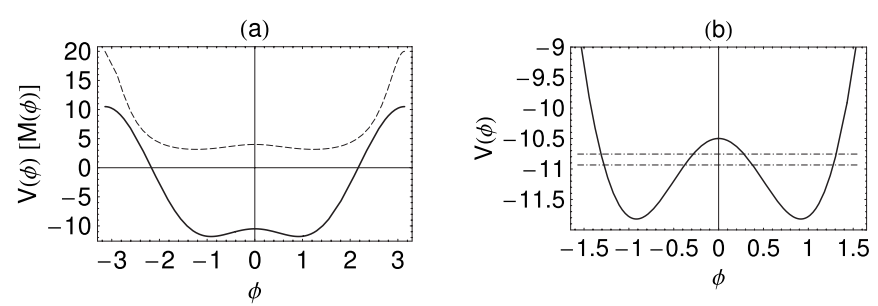

FIG. 3. (a) represents the plots of $V(\phi)$ (solid line) and $M(\phi)$ (dashed line) versus $\phi \in[-\pi, \pi]$ with $N_{p}=20$ and $\chi=1.5$ fixed. Note that both curves have similar behaviors and share complementary information about important quantum effects such as that appearing in (b), where now the energy levels $E_{0} / \epsilon$ and $E_{1} / \epsilon$ have also been drawn. The possibility of tunneling through the potential barrier can be blocked if the effective mass function presents a divergent behavior at or near the classical turning point; otherwise, the tunneling effect takes place (see both pictures at $\phi=0$ ). Such description of spin tunneling, via potential and effective mass functions, can also be extended to magnetic molecules, such as $\mathrm{Mn}_{12}$-acetate molecule and $\mathrm{Fe}_{8}$ clusters.

$\chi=1.5$ fixed. Note that the entropy functional curve presents an oscillatory behavior with a well-defined periodic structure, which allows us to estimate the energy gap through the period of oscillation between two consecutive maximum (minimum) points. Thus, after a detailed analysis of the numerical data used in the plot of Fig. 2, we can conclude that $\Delta \approx 0.1784$. It is important to emphasize that this result is in perfect agreement with that obtained from the diagonalization process for $\mathbf{H}_{L}$ (in this case, the percent error estimated is $\delta \approx 0.22 \%$ ).

Finally, we will present some plausible arguments that lead us to establish, under certain circumstances, a link between the oscillations of the discrete Husimi function, the energy gap, and the spin-tunneling effect. For this intent, let us initially adopt the theoretical framework exposed in Ref. [3], where the potential function

$$
V(\phi)=-\frac{N_{p}+1}{2}\left[\cos (\phi)+\frac{\chi}{2} \frac{N_{p}+3}{N_{p}+1} \sin ^{2}(\phi)\right]
$$

and the effective mass function

$$
M^{-1}(\phi)=\frac{2}{N_{p}-1}\left\{\cos (\phi)+\chi\left[1+\sin ^{2}(\phi)\right]\right\}
$$

were derived with details and exhaustively tested for the LMG model in the special limit $N_{p} \gg 1$. Such functions, defined in the interval $\phi \in[-\pi, \pi]$, permit us to explain, from a phenomenological point of view, the underlying behavior of the discrete Husimi function observed in Fig. 1. For instance, Fig. 3(a) shows the plots of $V(\phi)$ (solid line) and $M(\phi)$ (dashed line) versus $\phi$ for $N_{p}=20$ and $\chi=1.5$ fixed; in addition, Fig. 3(b) represents an amplified image of the curve related to $V(\phi)$, where now the lowest energy levels $E_{0} / \epsilon$ $=-10.9343$ and $E_{1} / \epsilon=-10.7555$, extracted from the diagonalization process of the Hamiltonian $\mathbf{H}_{L}$ in the $\mathbf{J}_{3}$ basis, are also viewed (see dotted-dashed lines). It should be stressed that distinct values of $\chi$ modify not only the energy gap but also the behavior of the curves related to the potential and effective mass functions. Hence, the value here chosen for $\chi$ brings out some explicit advantages of this theoretical approach since the potential function presents a pronounced barrier at the origin which affects, consequently, the energy levels $E_{0}$ and $E_{1}$. Indeed, Fig. 3(b) consists of a paradigmatic case where tunneling effects take place in this quasispin system. Within this context, Figs. 1(a) and 1(c) would then correspond to a spin wave packet equally distributed in both sides of the symmetric double-well potential, while Figs. 1(b) and 1(d) represent a wave packet localized only on one side of the potential barrier centered at $\phi=0$. Therefore, the time evolution of $\mathcal{H}(m, n ; \tau)$ can now also be understood as a faithful representation upon a discrete $N^{2}$-dimensional phase space (here labeled by the dimensionless angular momentum and angle pair) of spin-tunneling processes associated with the symmetric combination of the ground and first excited states.

\section{CONCLUDING REMARKS}

In what concerns the symmetry property related to the energy spectrum of the LMG Hamiltonian, let us mention some few words about the lowest energy doublet. Numerical investigations have shown that, if one considers the energy symmetric counterpart of this doublet, the time evolution of the spin wave packet will reveal a pronounced oscillatory behavior, and this result is directly associated with the high energetic demand of the system in accessing these particular states. The smoothing process due to Eq. (3) and the subsequent diagonalization of the discrete Husimi function will then produce, for all $\tau \geqslant 0$, an entropy functional $E\left[\left\{\lambda_{i}\right\} ; \tau\right]$ with a well-defined periodic pattern but not related to the previously discussed tunneling effects. Therefore, the theoretical apparatus here exposed shows that the coherent oscillations verified in this case do not characterize the same spintunneling process, although the value of the energy gap is the same.

Next, we introduce a complementary functional to that established by $E\left[\left\{\lambda_{i}\right\} ; t\right]$, which permits us to measure, in principle, the correlation between the discrete variables of an $N^{2}$-dimensional phase space. To this end, one considers the mutual correlation functional $[9,22]$

$$
I[\mathcal{H} ; t]:=E[\mathcal{Q} ; t]+E[\mathcal{R} ; t]-E[\mathcal{H} ; t] \geqslant 0,
$$

where $E[\mathcal{H} ; t]$ corresponds to the time-dependent joint entropy defined in terms of the discrete Husimi function (3), with $E[\mathcal{Q} ; t]$ and $E[\mathcal{R} ; t]$ representing the marginal entropies which are related to the respective marginal distributions $\mathcal{Q}(\mu ; t)$ and $\mathcal{R}(\nu ; t)$ (for technical details, see Refs. $[8,9]$ ). Thus, if one applies this measure to the LMG model, some interesting results can be promptly obtained. In this sense, Fig. 4 illustrates the time evolution of $I[\mathcal{H} ; \tau]$ versus $\tau$ $\in[0,60]$ for the same set of parameters fixed in the previous figures. From the numerical point of view, it is immediate to perceive the following: (i) The maximum points coincide with the configurations exhibited in Figs. 1(a) and 1(c) and also reflect a situation where the spin wave packet is equally distributed in both sides of the potential barrier, which implies in a minimal mutual correlation (maximal uncertainty) 


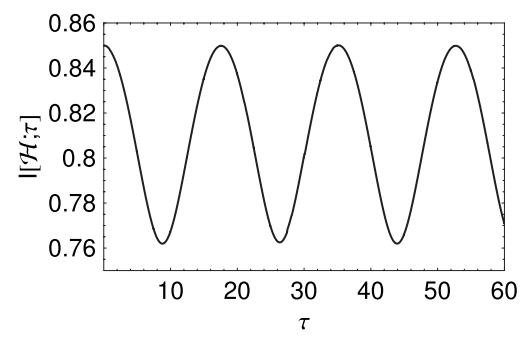

FIG. 4. Plot of $I[\mathcal{H} ; \tau]$ versus $\tau \in[0,60]$ for $N_{p}=20$ and $\chi$ $=1.5$ fixed. The oscillatory pattern viewed in this picture also allows us to estimate the energy gap from our numerical data, i.e., $\Delta$ $\approx 0.1786$, with an error of $\delta \approx 0.11 \%$.

between the angular momentum and angle pair; (ii) the minimum points describe both the configurations illustrated in Figs. 1(b) and 1(d) and this result can be explained by means of a spin wave packet localized in one of the potential wells, which leads us to obtain a maximal mutual correlation (minimal uncertainty) between the discrete variables $m$ and $n$; and finally, (iii) $I[\mathcal{H} ; \tau]$ presents a half-period if one compares with the oscillatory pattern exhibited by $E\left[\left\{\lambda_{i}\right\} ; \tau\right]$, this fact being associated with the absence of differentiation between the cases reported in Figs. 1(b) and 1(d).

In summary, we have developed an alternative theoretical framework for a class of physical systems described by discrete variables with potential applications in quantuminformation theory and quantum computation [23]. Based on a finite-dimensional phase-space description, this formalism was then applied to the Lipkin-Meshkov-Glick model [11] whose Hamiltonian operator, although apparently simple, presents some remarkable physical and mathematical properties [13]. In particular, we have shown how the angle-based potential approach [15-17] can be used to explain qualitatively the spin-tunneling effects related to a symmetric combination of ground and first excited states. Moreover, we have also inferred as a by-product the energy gap for this situation (i.e., related to the particular spin-tunneling situa- tion described here) through two different ways, and showed that both methods produce excellent quantitative results if one compares them with the exact analogues extracted from the diagonalization process of the LMG Hamiltonian.

Finally, it is worth mentioning that an interesting study about $\mathrm{Fe}_{8}$ magnetic clusters in the presence of external magnetic fields appeared recently in [24], where the theoretical apparatus for finite-dimensional phase spaces here discussed was extensively applied with great success in exploring the spin-tunneling effects in those clusters (in particular, such quantum effects occur for temperatures below the crossover temperature, i.e., $0.35 \mathrm{~K}$ [25]). Since the $\mathrm{Fe}_{8}$ magnetic clusters are characterized by a $J=10$ spin ground state [26], some similarities with the LMG model can be shared via discrete phase-space approach. However, it must be stressed that such phenomenological Hamiltonian, describing the $\mathrm{Fe}_{8}$ magnetic clusters, contains essential experimental information concerning the anisotropies inherent to the molecule structure [27], what produces an energy spectrum that is essentially diferent from that obtained for the LMG model. From this theoretical approach, it was also possible to infer the energy gap between the ground and first excited states, namely, $\Delta$ $\approx 2.92241 \mathrm{~K}$, with a percent error $\delta$ estimated in $1.04 \%$ (in this case, the external magnetic field applied along the easy axis of the cluster has as intensity $H_{\|}=0.11 \mathrm{~T}$ ). Besides, the entropy functionals $E[\mathcal{H} ; \tau]$ and $I[\mathcal{H} ; \tau]$ were also employed to qualitatively explain how the spin-tunneling effect is connected with the functional correlations between the discrete variables observed in the underlying phase space, corroborating, in this way, the results here presented.

\section{ACKNOWLEDGMENTS}

The authors thank Paulo E. M. F. Mendonça from the University of Queensland (Australia) for providing valuable suggestions, and two anonymous referees for useful comments on an earlier version of this paper. This work has been supported by CAPES and CNPq, both Brazilian agencies for financial support.
[1] E. M. Chudnovsky and J. Tejada, Macroscopic Quantum Tunneling of the Magnetic Moment (Cambridge University Press, Cambridge, UK, 1998); S. Takagi, Macroscopic Quantum Tunneling (Cambridge University Press, Cambridge, UK, 2002).

[2] J. L. van Hemmem and A. Süto, Europhys. Lett. 1, 481 (1986); M. Enz and R. Schilling, J. Phys. C 19, 1765 1986; G. Scharf, W. F. Wreszinski, and J. L. van Hemmem, J. Phys. A 20, 4309 (1987); O. B. Zaslavskii, J. Phys.: Condens. Matter 1, 6311 (1989); V. V. Ulyanov and O. B. Zaslavskii, Phys. Rep. 216, 179 (1992).

[3] D. Galetti and A. F. R. de Toledo Piza, Physica A 149, 267 (1988).

[4] W. K. Wootters, Ann. Phys. (N.Y.) 176, 1 (1987); O. Cohendet, P. Combe, M. Sirugue, and M. Sirugue-Collin, J. Phys. A 21, 2875 (1988); P. Leboeuf and A. Voros, ibid. 23, 1765 (1990); D. Galetti and A. F. R. de Toledo Piza, Physica A 186, 513 (1992); T. Opatrný, D.-G. Welsch, and V. Bužek, Phys.
Rev. A 53, 3822 (1996); A. Luis and J. Peřina, J. Phys. A 31, 1423 (1998); T. Hakioglu, ibid. 31, 6975 (1998); A. Takami, T. Hashimoto, M. Horibe, and A. Hayashi, Phys. Rev. A 64, 032114 (2001); N. Mukunda, S. Chatuverdi, and R. Simon, Phys. Lett. A 321, 160 (2004); A. Vourdas, Rep. Prog. Phys. 67, 267 (2004); K. S. Gibbons, M. J. Hoffman, and W. K. Wootters, Phys. Rev. A 70, 062101 (2004); A. B. Klimov, L. L. Sánchez-Soto, and H. de Guise, J. Phys. A 38, 2747 (2005); A. B. Klimov, C. Muñoz, and J. L. Romero, ibid. 39, 14471 (2006); A. Vourdas, ibid. 39, R65 (2006).

[5] D. Galetti and M. A. Marchiolli, Ann. Phys. (N.Y.) 249, 454 (1996).

[6] D. Galetti and M. Ruzzi, Physica A 264, 473 (1999).

[7] M. Ruzzi, M. A. Marchiolli, and D. Galetti, J. Phys. A 38, 6239 (2005).

[8] M. A. Marchiolli, M. Ruzzi, and D. Galetti, Phys. Rev. A 72, 042308 (2005). 
[9] M. A. Marchiolli, M. Ruzzi, and D. Galetti, Phys. Rev. A 76, 032102 (2007).

[10] J. Schwinger, Advanced Book Classics: Quantum Kinematics and Dynamics (Addison-Wesley, New York, 1991); Quantum Mechanics: Symbolism of Atomic Measurements (SpringerVerlag, Berlin, 2001).

[11] H. J. Lipkin, N. Meshkov, and A. J. Glick, Nucl. Phys. 62, 188 (1965); N. Meshkov, and A. J. Glick, and H. J. Lipkin, ibid. 62, 199 (1965); A. J. Glick, H. J. Lipkin, and N. Meshkov ibid. 62, 211 (1965).

[12] P. Ring and P. Schuck, The Nuclear Many-Body Problem (Springer-Verlag, Berlin, 2004).

[13] R. Shankar, Phys. Rev. Lett. 45, 1088 (1980); A. Klein and E. R. Marshalek, Rev. Mod. Phys. 63, 375 (1991); J. Vidal, G. Palacios, and R. Mosseri, Phys. Rev. A 69, 022107 (2004); A. Garg and M. Stone, Phys. Rev. Lett. 92, 010401 (2004); T. Barthel, S. Dusuel, and J. Vidal, ibid. 97, 220402 (2006); P. Ribeiro, J. Vidal, and R. Mosseri, ibid. 99, 050402 (2007); G. Rosensteel, D. J. Rowe, and S. Y. Ho, J. Phys. A 41, 025208 (2008); P. K. Pathak, R. N. Deb, N. Nayak, and B. Dutta-Roy, ibid. 41, 145302 (2008); P. Solinas, P. Ribeiro, and R. Mosseri, Phys. Rev. A 78, 052329 (2008).

[14] S. Dusuel and J. Vidal, Phys. Rev. B 71, 224420 (2005).

[15] D. Galetti and M. Ruzzi, J. Phys. A 33, 2799 (2000).

[16] D. Galetti, B. M. Pimentel, and C. L. Lima, Physica A 351, 315 (2005).

[17] D. Galetti, Physica A 374, 211 (2007).

[18] D. Galetti and E. C. Silva, Physica A 386, 219 (2007).

[19] K. E. Cahill and R. J. Glauber, Phys. Rev. 177, 1857 (1969); 177, 1882 (1969).

[20] R. A. Horn and C. R. Johnson, Matrix Analysis (Cambridge
University Press, Cambridge, UK, 1985); R. Aldrovandi, Special Matrices of Mathematical Physics: Stochastic, Circulant and Bell Matrices (World Scientific, Singapore, 2001); F. R. Gantmacher, Applications of the Theory of Matrices (Dover, New York, 2005).

[21] In fact, it is a subspace of the full Hilbert space related to the LMG model, where the Liouvillian dynamics does not access any adjacent multiplets for all $t \geqslant 0$. Hence, all the physical information necessary to describe any quantum effects associated with spin tunneling and/or correlations is completely restricted to a particular block of the Hamiltonian matrix responsible for the ground state.

[22] A. Wehrl, Rev. Mod. Phys. 50, 221 (1978); V. Vedral, ibid. 74, 197 (2002); J. Audretsch, Entangled Systems: New Directions in Quantum Physics (Wiley-VCH, Berlin, 2007).

[23] M. A. Nielsen and I. L. Chuang, Quantum Computation and Quantum Information (Cambridge University Press, Cambridge, UK, 2000); I. Bengtsson and K. Życzkowski, Geometry of Quantum States: An Introduction to Quantum Entanglement (Cambridge University Press, Cambridge, UK, 2008).

[24] E. C. Silva and D. Galetti, e-print arXiv:0806.4105v2.

[25] C. Sangregorio, T. Ohm, C. Paulsen, R. Sessoli, and D. Gatteschi, Phys. Rev. Lett. 78, 4645 (1997).

[26] Some important experimental data related to the $\mathrm{Fe}_{8}$ magnetic cluster indicate that such physical system has a $J=10$ spin, which implies a 21-dimensional state space for this particular degree of freedom, with an observed potential barrier of about $24 \mathrm{~K}$.

[27] A. Caneschi, D. Gatteschi, C. Sangregorio, R. Sessoli, L. Sorace, A. Cornia, M. A. Novak, C. Paulsen, and W. Wernsdorfer, J. Magn. Magn. Mater. 200, 182 (1999). 